\title{
Characteristic Curves of the Lennard-Jones Fluid
}

\author{
Simon Stephan ${ }^{1}$ D . Ulrich K. Deiters ${ }^{2}$
}

Received: 21 May 2020 / Accepted: 25 July 2020 / Published online: 20 August 2020

(c) The Author(s) 2020

\begin{abstract}
Equations of state based on intermolecular potentials are often developed about the Lennard-Jones (LJ) potential. Many of such EOS have been proposed in the past. In this work, $20 \mathrm{LJ}$ EOS were examined regarding their performance on Brown's characteristic curves and characteristic state points. Brown's characteristic curves are directly related to the virial coefficients at specific state points, which can be computed exactly from the intermolecular potential. Therefore, also the second and third virial coefficient of the LJ fluid were investigated. This approach allows a comparison of available LJ EOS at extreme conditions. Physically based, empirical, and semi-theoretical LJ EOS were examined. Most investigated LJ EOS exhibit some unphysical artifacts.
\end{abstract}

Keywords Lennard-Jones fluid - Equation of state - Characteristic curves · Virial coefficients

\section{Introduction}

The Lennard-Jones $(12,6)$ potential $[1,2]$ has been extensively used since the early days of computer simulation [3-6] for the modeling of repulsive and dispersive interactions of simple fluids. It is probably the most frequently investigated monomer model fluid in molecular simulation [7]. The Lennard-Jones (LJ) potential can be favorably used for testing new theories and simulation methods, e.g., for mixtures, phase changes, non-equilibrium phenomena, and interfaces between phases

Electronic supplementary material The online version of this article (https://doi.org/10.1007/s1076 5-020-02721-9) contains supplementary material, which is available to authorized users.

Simon Stephan

simon.stephan@mv.uni-kl.de

1 Laboratory of Engineering Thermodynamics (LTD), TU Kaiserslautern, Erwin-Schrödinger-Straße 44, 67663 Kaiserslautern, Germany

2 Institute of Physical Chemistry, University of Cologne, Greinstraße 4-6, 50939 Cologne, Germany 
[8-19]. Also, the Lennard-Jones potential is often used as a starting point for the development of many state-of-the-art force fields for complex molecules [20-22].

The Lennard-Jones potential is defined as

$$
u_{\mathrm{LJ}}(r)=4 \varepsilon\left[\left(\frac{\sigma}{r}\right)^{12}-\left(\frac{\sigma}{r}\right)^{6}\right],
$$

where $\varepsilon$ and $\sigma$ are the energy and size parameter, respectively. The distance between two particles is denoted by $r$. Different versions of the LJ potential are used in the literature depending on the treatment of the long-range interactions, which has an important influence on the thermodynamic properties [23-32]. The present work is limited to the 'full' Lennard-Jones potential, i.e., including long-range correction schemes [33].

Analytical model functions of the LJ fluid for the description of the thermodynamic properties, i.e., equations of state (EOS), are crucial for many applications, e.g., the development of theories for more complex fluids like polymers, electrolyte solutions, and associating fluids. LJ EOS have been used successfully as base models for a reference fluid to describe more complex fluids [34-38]. A large number of equations of state have been proposed for the LJ fluid, of which 20 are studied here, cf. Table 1.

Table 1 Lennard-Jones equations of state used in the present work-sorted chronologically

\begin{tabular}{llll}
\hline Authors & Abbr. & EOS type & Year \\
\hline Nicolas et al. [51] & Ni & Empirical (MBWR); pressure explicit & 1979 \\
Ree [53] & Re & Empirical; pressure explicit & 1980 \\
Cotterman et al. [35] & Co & BH perturbation theory + virial; Helmholtz energy explicit & 1986 \\
Adachi et al. [64] & Ad & Empirical (MBWR); pressure explicit & 1988 \\
Koutras et al. [66] & Kou & Modified HS equation; pressure explicit & 1992 \\
Miyano [61] & Mi & Empirical (MBWR); pressure explicit & 1993 \\
Johnson et al. [28] & Jo & Empirical (MBWR); pressure explicit & 1993 \\
Kolafa and Nezbeda [52] & Ko & HS + virial + empirical; Helmholtz energy explicit & 1994 \\
Mecke et al. [54, 55] & Me & HS + empirical; Helmholtz energy explicit & 1996 \\
Sun and Teja [60] & Su & Empirical (MBWR); pressure explicit & 1996 \\
Hess [67] & He & WCA reference + virial; pressure explicit & 1999 \\
Boltachev and Baidakov [68] & Bo & Empirical + virial; pressure explicit & 2003 \\
Paricaud [57] & Pa & BH perturbation theory; Helmholtz energy explicit & 2006 \\
Quiñones-Cisneros et al. [65] & Qui & Empirical; pressure explicit & 2009 \\
May and Mausbach [62, 63] & Ma & Empirical (MBWR); pressure explicit \\
Lafitte et al. [38] & La & BH perturbation theory; Helmholtz energy explicit & 2012 \\
Thol et al. [41] & Th & Empirical; Helmholtz energy explicit & 2013 \\
van Westen and Gross [56] & vWe & BH perturbation theory; Helmholtz energy explicit & 2016 \\
Gottschalk [69] & Go & Empirical; virial coefficients; Helmholtz energy explicit & 2019 \\
Stephan et al. [46] & St & BH perturbation theory; Helmholtz energy explicit & 2020 \\
\hline
\end{tabular}

The columns are: authors, abbreviation for EOS, EOS type, and year of publication 
Lennard-Jones EOS can be broadly separated into two types: empirical EOS and physically motivated EOS. 'Physically motivated' EOS means that the employed functions were derived from statistical mechanics, whereas an empirical EOS is a correlation of computer experiment data by a convenient but arbitrary mathematical form. However, the distinction between both types is not sharp; most LJ EOS have some physically motivated features and some empirical features, cf. Deiters and de Reuck [39] for a detailed discussion. It is widely accepted that empirical EOS have a tendency to exhibit poor extrapolation behavior to fluid regions and physical properties that were not considered during the parametrization and may even yield a physically unreasonable behavior in some regions, e.g., multiple van der Waals loops in the vapor-liquid two phase region [40-42] or the crossing of isotherms [43-45]. Physically motivated LJ EOS on the other side are often less precise in the description of homogeneous state properties-particularly higher-order thermodynamic derivatives.

The LJ EOS of Johnson et al. [28], Lafitte et al. [38], and Stephan et al. [46] are of particular interest since those are the base in SAFT [34, 47, 48] type EOS for the modeling of repulsive and dispersive interactions, i.e., soft-SAFT [36, 37], SAFT-VR Mie [38, 49], and PC-SAFT [50], respectively. The LJ EOS of Ref. [46] is a re-parametrization of the PC-SAFT monomer model—developed to give a good description of the LJ fluid.

The most popular LJ EOS are those of Johnson et al. [28], Nicolas et al. [51], Kolafa and Nezbeda [52], Cotterman et al. [35], Lafitte et al. [38], Ree [53], and Mecke et al. [54,55] (sorted by their the number of citations in the Web of Science).

Table 1 also indicates the types of the LJ EOS, i.e., whether they are Helmholtz energy or pressure explicit. We compare the performance of 20 LJ EOS, varying from purely empirical to rigorously theory-based. Physically motivated LJ EOS considered here are those of Refs. [35, 38, 46, 56, 57] and are all based on the perturbation theory of Barker and Henderson [58, 59]. LJ EOS that are empirically based are those of Refs. [28, 41, 51, 53, 60-65]. The remaining LJ EOS considered in the present work [52, 54, 55, 66-69] are denoted here as semi-theoretical.

In a recent work of our group [46], LJ EOS were systematically reviewed and compared with available computer experiment data for the LJ fluid for homogeneous state points and the vapor-liquid equilibrium. The present work pursues this comparison for a detailed discussion of Brown's characteristic curves [70] and important characteristic states. As Brown's characteristic curves are directly related to virial coefficients, also the second and third virial coefficient are studied. This comparison is of particular interest, since the virial coefficients of the LJ fluid can be computed exactly from their definitions in statistical mechanics, while reference data obtained from computer simulations are subject to errors and uncertainties [7, 71, 72]. Brown's characteristic curves and the virial coefficients are directly linked in the limit of the ideal gas and therefore corporately investigated in the present work. Brown proposed the characteristic curves for the assessment of equations of state for a fluid with repulsive and dispersive interactions [70]. The LJ fluid is evidently an excellent candidate for such an assessment. Furthermore, Brown's characteristic curves are an important tool for the development of new equations of state [73-75]. 
From Brown's characteristic curves, the Amagat curve exhibits the largest pressure and temperature. For most gases, the Amagat curve is therefore not relevant for technical applications. Nevertheless, for particularly light-boiling gases, like neon, helium, and hydrogen, the pressure and temperature range of the Amagat curve is often relevant for technical applications. Furthermore, thermodynamic conditions in the range of the Amagat curve are relevant for fluids in geological applications as well as lubrication gaps in tribological applications. Only EOS that produce reasonable Amagat curves are appropriate for such applications.

We use reduced properties with respect to the Lennard-Jones potential throughout this article denoted by an asterisk; the definitions can be found in Table 2.

\section{Theory}

Brown's characteristic curves are defined as curves on which the compressibility factor $Z=\frac{p^{*} v^{*}}{T^{*}}$ or its derivatives match the values of an ideal gas at the same temperature and density [70]. Since EOS are usually fitted to reference data at moderate conditions, the application of the characteristic curves is often referred to as 'testing the extrapolation behavior' of EOS [41, 73-75]. The testing of these characteristic curves has also been included in the IUPAC guidelines for publishing equations of state [76]. In this section, the definitions of Brown's characteristic curves along with the description of the their general features are briefly outlined. Also their relation to the second and third virial coefficient $B^{*}$ and $C^{*}$, respectively, is discussed.

Brown's characteristic curves are defined as the loci of state points at which a certain thermodynamic property of the fluid matches that of an ideal gas $[70,73,75,77]$. Brown defined four main characteristic curves: one $0^{\text {th }}$-order (named Zeno curve) and three $1^{\text {st }}$-order curves (named Amagat, Boyle, and Charles curve) [70] based on the compressibility factor itself and its derivatives with respect to the temperature and

Table 2 Reduced properties with respect to the LennardJones potential parameters $\varepsilon$ and $\sigma$ applied in the present work

\begin{tabular}{ll}
\hline Property & Symbol \\
\hline Temperature & $T^{*}=T /\left(\varepsilon / k_{\mathrm{B}}\right)$ \\
Pressure & $p^{*}=p /\left(\varepsilon / \sigma^{3}\right)$ \\
Density & $\rho^{*}=\rho /\left(1 / \sigma^{3}\right)$ \\
Internal energy & $u^{*}=u / \varepsilon$ \\
Helmholtz energy & $a^{*}=a / \varepsilon$ \\
Enthalpy & $h^{*}=h / \varepsilon$ \\
Distance & $r^{*}=r / \sigma$ \\
Volume & $V^{*}=V / \sigma^{3}$ \\
Specific volume & $v^{*}=v / \sigma^{3}$ \\
$2^{\text {nd }}$ virial coeff. & $B^{*}=B /\left(\frac{2}{3} \pi \sigma^{3}\right)$ \\
$3^{\text {rd }}$ virial coeff. & $C^{*}=C /\left(\frac{2}{3} \pi \sigma^{3}\right)^{2}$ \\
\hline
\end{tabular}

The properties $u^{*}, a^{*}, h^{*}, v^{*}$ indicate the respective quantity per particle 
pressure. For a real fluid, $Z$ and its derivatives can match the values of the ideal gas for special $T^{*}, v^{*}$ combinations only $[70,75]$, as a result of Gibbs' phase rule. These state points collectively constitute a characteristic curve.

The characteristic curves are also known under other names: the Zeno curve as 'ideal curve', the Amagat curve as 'Joule inversion curve', and the Charles curve as 'Joule-Thomson inversion curve'. Here, we adopt the original naming introduced by Brown [70]. The characteristic curves are usually plotted in a double-logarithmic $p^{*}$ $-T^{*}$ diagram; this convention is also adopted here. In such a diagram, the characteristic curves exhibit a typical concave dome shape, i.e., they have a negative curvature.

The Zeno, Amagat, Boyle, and Charles curve are required to have a negative curvature throughout and a single maximum in a double-logarithmic pressure-temperature diagram [70, 75]. Furthermore, Brown postulated that all four characteristic curves end in the limit of $p^{*} \rightarrow 0$ with an infinite slope in a double-logarithmic $p^{*}-T^{*}$ diagram [70]. Brown furthermore deduced that the four characteristic curves of $0^{\text {th }}$ and $1^{\text {st }}$-order may only contact each other at three distinct points [70, 75]: (1) the Zeno curve converges against the Amagat curve on the hypothetical extension of the vapor pressure curve; (2) the Zeno curve converges against the Boyle curve in their common limit of $p^{*} \rightarrow 0$ at $T^{*}=T_{\text {Boyle }}^{*}$ (the zero crossing temperature of the second virial coefficient), and (3) the Zeno curve intersects the Charles curve at the point of maximum pressure of the Zeno curve. Usually, the Amagat and Zeno curves are truncated at low temperatures by the solid-fluid equilibrium. Finally, the Amagat, Boyle, and Charles curve must not cross, but enclose each other in a $p^{*}-T^{*}$ diagram [70]: the Amagat curve surrounding the Charles curve surrounding the Boyle curve.

The characteristic curves can be computed from the Helmholtz energy per particle $a^{*}$ and its derivatives. The following notation is used for the derivatives of the Helmholtz energy with respect to the inverse temperature and density

$$
\tilde{a}_{n m}^{*}=\tilde{a}_{n m}^{* \mathrm{id}}+\tilde{a}_{n m}^{* \operatorname{conf}}=\left(1 / T^{*}\right)^{n} \rho^{* m} \frac{\partial^{n+m}\left(\tilde{a}^{* \mathrm{id}}+\tilde{a}^{* \mathrm{conf}}\right)}{\partial\left(1 / T^{*}\right)^{n} \partial \rho^{* m}},
$$

with $n, m=0,1,2$ and the tilde indicating $\tilde{a}^{*}=a^{*} / T^{*}$. In Eq. (2), 'id' indicates the ideal gas contribution and 'conf' the configurational contribution.

The density-based virial equation can be written as

$$
Z=1+B^{*} \rho^{*}+C^{*} \rho^{* 2}+\cdots .
$$

The Zeno curve $(\mathcal{Z})$ is defined as the locus of state points that satisfy $Z=\frac{p^{*} v^{*}}{T^{*}} \equiv 1$ and can be computed from the Helmholtz energy as

$$
\tilde{a}_{01}^{* \operatorname{conf}}=0 \text {. }
$$

Furthermore, state points on the Zeno curve have $u^{* \text { conf }}=0$. The Zeno curve ends at the Boyle temperature $T_{\text {Boyle }}^{*}$ in the zero-pressure limit $p^{*} \rightarrow 0$, where the third and higher virial coefficients can be neglected. This corresponds to the condition for the second virial coefficient $B^{*}\left(T_{\text {Boyle }}^{*}\right)=0$, cf. Eq. (3).

The Amagat curve $(\mathcal{A})$ is defined as the locus of state points that satisfy $\left(\frac{\partial Z}{\partial T^{*}}\right)_{v^{*}} \equiv 0$ and can be computed from the Helmholtz energy as 


$$
\tilde{a}_{11}^{* \operatorname{conf}}=0 .
$$

The Amagat curve originates on the vapor pressure curve at low temperatures (if crystallization is disregarded). It ends at the Amagat temperature $T_{\text {Amagat }}^{*}$ in the zeropressure limit $p^{*} \rightarrow 0$, which corresponds to the maximum of the second virial coefficient with $\mathrm{d} B^{*} / \mathrm{d} T^{*}=0$. This relation can be obtained by applying $\left(\frac{\partial Z}{\partial T^{*}}\right)_{v^{*}}=0$ to the density-based virial equation (3) in the low-pressure limit where the third and higher virial coefficients can be neglected.

Equations of state that do not exhibit a maximum in the second virial coefficient $B^{*}\left(T^{*}\right)$ have a distorted Amagat curve in the limit $p^{*} \rightarrow 0[70,75]$.

The Boyle curve $(\mathcal{B})$ is defined as the locus of state points that satisfy $\left(\frac{\partial Z}{\partial 1 / \rho^{*}}\right)_{T^{*}} \equiv 0$ and can be computed from the Helmholtz energy as

$$
\tilde{a}_{01}^{* \operatorname{conf}}+\rho^{*} \tilde{a}_{02}^{* \operatorname{conf}}=0 .
$$

The Boyle curve originates on the vapor pressure curve close to the critical point, runs through a pressure maximum and ends at the Boyle temperature in the limit $p^{*} \rightarrow 0$. This can be shown by applying $\left(\frac{\partial Z}{\partial 1 / \rho^{*}}\right)_{T^{*}}=0$ to Eq. (3) in the low-pressure limit. Hence, the Boyle and the Zeno curve converge into each other in the zeropressure limit.

The Charles curve $(\mathcal{C})$ is defined as the locus of state points that satisfy $\left(\frac{\partial Z}{\partial T^{*}}\right)_{p^{*}} \equiv 0$ and can be computed from the Helmholtz energy as

$$
\tilde{a}_{01}^{* \operatorname{conf}}+\rho^{*} \tilde{a}_{02}^{* \operatorname{conf}}+1 / T^{*} \tilde{a}_{11}^{* \operatorname{conf}}=0 .
$$

The Charles curve-also known as Joule-Thomson inversion curve-is of fundamental technical importance as it determines the transition locus from heating to cooling upon isenthalpic throttling, i.e., $\left(\frac{\partial T^{*}}{\partial p^{*}}\right)_{h^{*}}=0$ also holds on the Charles curve. The Charles curve originates on the vapor pressure curve. The Charles curve ends at the Charles temperature $T_{\text {Charles }}^{*}$ in the zero-pressure limit $p^{*} \rightarrow 0$, which corresponds to the condition for the second virial coefficient $\mathrm{d} B^{*} / \mathrm{d} T^{*}=B^{*} / T^{*}$ (the secant of the second virial coefficient at $B^{*}\left(T_{\text {Charles }}^{*}\right)$ is a line through the origin) [39]. This relation can be straightforwardly derived by applying $\left(\frac{\partial Z}{\partial T^{*}}\right)_{p^{*}}=0$ to the pressure-based virial equation in the low-density limit where the third and higher virial coefficients are negligible [74].

Furthermore, it has been shown that the terminal slope of the characteristic curves at high temperatures are related to both the second and third virial coefficient [74], which follows from the nature of the virial expansion.

Details and alternative thermodynamic definitions for the characteristic curves can be found in Refs. [39, 70, 73, 75, 77].

The second and third virial coefficient $B^{*}$ and $C^{*}$, respectively, of a fluid can be directly computed from the pairwise additive intermolecular potential, e.g., the LJ potential $u_{\mathrm{LJ}}[78,79]$. Using the Mayer function

$$
f_{i j}^{*}=\exp \left(-u_{\mathrm{LJ}}^{*}\left(r_{i j}^{*}\right) / T^{*}\right)-1,
$$


where $r_{i j}^{*}$ indicates the distance between two interacting particles, the second and third virial coefficient can be written as [79]

$$
\begin{gathered}
B^{*}=-\frac{1}{2 V^{*}} \iint f_{12}^{*} \mathrm{~d} \mathbf{r}_{1}^{*} \mathrm{~d} \mathbf{r}_{2}^{*}, \\
C^{*}=-\frac{1}{3 V^{*}} \iiint f_{12}^{*} f_{23}^{*} f_{13}^{*} \mathrm{~d} \mathbf{r}_{1}^{*} \mathrm{~d} \mathbf{r}_{2}^{*} \mathrm{~d} \mathbf{r}_{3}^{*},
\end{gathered}
$$

where $\mathrm{d} \mathbf{r}^{*}$ indicates a finite volume element in which the particle is located. Equation (9) can be integrated in a trivial way, cf. Ref. [79]. The integrals in Eq. (10) were solved in this work using the method proposed by Hutem and Boonchui [80]. Equations (9) and (10) were implemented and numerically integrated to obtain exact results (within the significant digits used for the computer precision in the calculations) for $B^{*}$ and $C^{*}$ as well as their characteristic points using the LJ potential. 'Exact' means here that no statistical uncertainty applies to the data (in contrast to molecular simulation results).

Furthermore, the second and third viral coefficient were computed from the considered LJ EOS, cf. Table 1. Equations (11) and (12) give the thermodynamic definitions for the calculation of the second and third virial coefficient $B^{*}$ and $C^{*}$ from the Helmholtz energy:

$$
\begin{gathered}
B^{*}=\lim _{\rho^{*} \rightarrow 0}\left(\frac{\partial\left(p^{*} / \rho^{*} T^{*}\right)}{\partial \rho^{*}}\right)_{T^{*}}=\rho^{*-1} \lim _{\rho^{*} \rightarrow 0}\left(\tilde{a}_{01}^{* \operatorname{conf}} / \rho^{*}\right), \\
C^{*}=1 / 2 \cdot \lim _{\rho^{*} \rightarrow 0}\left(\frac{\partial^{2}\left(p^{*} / \rho^{*} T^{*}\right)}{\partial \rho^{* 2}}\right)_{T^{*}}=\rho^{*-2} \lim _{\rho^{*} \rightarrow 0}\left(\tilde{a}_{02}^{* \operatorname{conf}} / \rho^{* 2}\right) .
\end{gathered}
$$

\section{Results}

\subsection{Virial Coefficients}

The second and third virial coefficient computed from the 20 considered LJ EOS are compared in Fig. 1 with exact data obtained from statistical mechanics [81] published in the literature [7, 29, 60, 82-84]. Numbers from our implementation perfectly agree with that literature data. The literature values are plotted for reproducibility reasons. The numeric values for the second and third virial coefficient computed from the 20 considered LJ EOS are reported in the electronic Supplementary Material.

Qualitatively, the second virial coefficient $B^{*}$ of the LJ fluid is captured well by all considered LJ EOS, cf. Fig. 1-top, except that of Koutras et al. [66]. All other LJ EOS have a single zero crossing at the Boyle temperature $T_{\text {Boyle }}^{*}$ (defined as $\left.B^{*}\left(T_{\text {Boyle }}^{*}\right)=0\right)$. The exact value of the Boyle temperature obtained from numerical 

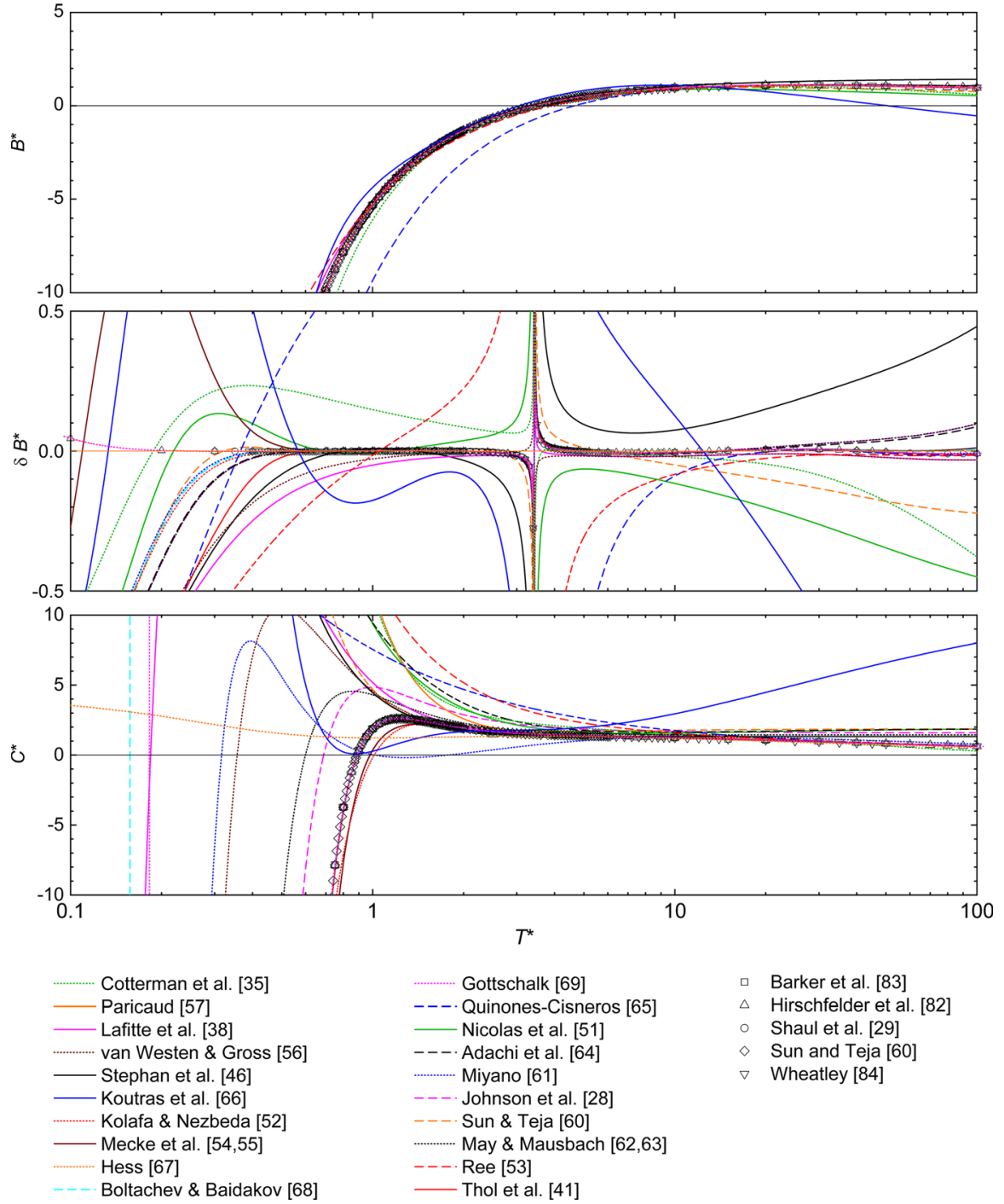

Fig. 1 Second (top and middle) and third (bottom) virial coefficient as a function of the temperature. The top and bottom plot show the virial coefficients themselves; the middle plot shows the relative deviation $\delta B^{*}=\left(B_{\mathrm{EOS}}^{*}-B_{\mathrm{ref}}^{*}\right) / B_{\text {ref }}^{*}$ of the second virial coefficient from the LJ EOS of Paricaud [57], i.e., the baseline 'ref' corresponds to Ref. [57]. For all three plots: lines are LJ EOS and exact values from Refs. [29, $60,82-84]$ are symbols

integration is $T_{\text {Boyle }}^{*}=3.417927982$. However, significant deviations from the exact second virial coefficient data are found for most LJ EOS at low temperatures (below the triple point temperature, which is approximately $T_{\mathrm{tr}}^{*}=0.68 \pm 0.02$ [85-92]) and at high temperatures $\left(T^{*}>10\right)$, cf. Fig. $1-$ middle. Excluding the direct vicinity of the Boyle temperature, all LJ EOS except that of Paricaud [57], Gottschalk [69], and 
Hess [67] show relative deviations from the exact values for the second virial coefficient of at least $20 \%$ in some temperature range. The LJ EOS of Refs. [57, 67, 69] comprise the statistical mechanical formulation for the second virial coefficient in their mathematical formulation, which consequently leads to an excellent agreement for $B^{*}$. However, minor deviations for $B^{*}$ are observed for the results obtained from our implementation of the LJ EOS of Paricaud [57] at low temperatures.

The LJ EOS of Quiñones-Cisneros et al. [65], Nicolas et al. [51], Cotterman et al. [35], Sun and Teja [60], Koutras et al. [66], and Stephan et al. [46] deviate by more than $20 \%$ from the exact values in the range $T^{*}<T_{\text {tr }}^{*}$ and $6<T^{*}$. The LJ EOS of van Westen and Gross [56], Lafitte et al. [38], Thol et al. [41], Adachi et al. [64], May and Mausbach [62, 63], Johnson et al. [28], Kolafa and Nezbeda [52], Boltachev and Baidakov [68], Ree [53], and Miyano [61] deviate by more than 20\% from the exact values at $T^{*}<T_{\text {tr }}^{*}$, too, but perform better at high temperatures. Excluding the vicinity of the Boyle temperature and extreme temperature conditions at $T^{*}<T_{\text {tr }}^{*}$ and $6<T^{*}$, the LJ EOS of Mecke et al. [54, 55], Johnson et al. [28], Boltachev and Baidakov [68], Adachi et al. [64], Miyano [61], Thol et al. [41], and Kolafa and Nezbeda [52] describe the exact second virial coefficient data within $\delta B^{*}= \pm 2 \%$ - the LJ EOS of Refs. $[41,52,54,55,64,68]$ even better than $0.5 \%$ in a wide temperature range.

The agreement of the LJ EOS and exact values for the third virial coefficient $C^{*}$ is overall significantly less good than for the second virial coefficient. Only the LJ EOS of Johnson et al. [28], Kolafa and Nezbeda [52], Lafitte et al. [38], Mecke et al. [54, 55], May and Mausbach [62, 63], Thol et al. [41], and van Westen and Gross [56] qualitatively describe the trend of the third virial coefficient accurately. The LJ EOS of Kolafa and Nezbeda [52], Mecke et al. [54, 55], and Thol et al. [41] describe the third virial coefficient qualitatively well up to $T^{*}=100$. The absolute average deviations from these three LJ EOS and the exact values for $C^{*}$ from the literature (Refs. $[7,29,60,82-84,93])$ are $\mathrm{AAD}^{\mathrm{Me}}=0.47, \mathrm{AAD}^{\mathrm{Ko}}=0.53$, and $\mathrm{AAD}^{\mathrm{Th}}=0.08$, i.e., that of Thol et al. [41] is the most accurate regarding the description of $C^{*}$. Also the results from the LJ EOS of Boltachev and Baidakov [68] and Gottschalk [69] are in very good agreement with the exact data for the third virial coefficient at moderate and high temperatures (cf. Fig. 1-bottom), too, but both yield a wrong limit at low temperatures.

Other considered LJ EOS either exhibit no maximum or two maxima or a wrong limit at low temperatures. Even though the LJ EOS of Refs. [57, 67, 69] were found to be the most precise LJ EOS to describe the second virial coefficient, these LJ EOS produce a qualitatively wrong trend for the third virial coefficient. As it is possible to discriminate between the LJ EOS based on their ability to reproduce $B^{*}\left(T^{*}\right)$ and particularly $C^{*}\left(T^{*}\right)$, no attempt was made to discuss their predictions of $D^{*}\left(T^{*}\right)$ and higher virial coefficients.

Castro-Marcano et al. [94] showed that theoretically based EOS, such as softSAFT [36, 37], SAFT-VR [38, 49], and PC-SAFT [50, 95] do not adequately describe third virial coefficients of real substances in a sense that they do not exhibit a maximum at moderate temperatures and wrong limits at low temperatures. Our results indicate that for the PC-SAFT equation, this deficiency is already inherent in 
the corresponding monomer term (the LJ EOS of Ref. [46]), whereas the soft-SAFT and SAFT-VR Mie equation show a physically correct behavior for monomers.

\subsection{Characteristic Curves}

Multiple computer experiment data sets for the characteristic curves of the LJ fluid are available in the literature [75, 96-100]. The Charles curve (a.k.a. Joule-Thomson inversion curve) of the LJ fluid has been investigated several times in the literature by molecular simulations [75, 96-100]. The Amagat, Boyle, and Zeno curve of the LJ fluid have only been investigated by computer experiment by Deiters and Neumaier [75]. The numeric values of these computer experiment data were summarized in Ref. [7] and are taken here as reference.

The computer experiment data available for the characteristic curves are compared in Fig. 2 with the results obtained from the LJ EOS of Lafitte et al. [38], which gives the best description of the characteristic curve reference data (discussed in detail below). The Charles curve computer experiment data points of Refs. [75, 96, 97, 99, 100] are in good mutual agreement. For the Charles curve, the computer experiment data reported by Heyes and Llaguno [98] is found to deviate significantly from the data of Refs. [75, 96, 97, 99, 100]. To avoid visual clutter, only the data of

Fig. 2 Brown's characteristic curves: lines are the LJ EOS of Lafitte et al. [38]; symbols are molecular simulations results from the literature [75, 96-100]. The black solid line and star indicate the VLE and critical point. The gray-shaded region indicates the solid phase of the LJ potential as reported by Agrawal and Kofke [89]

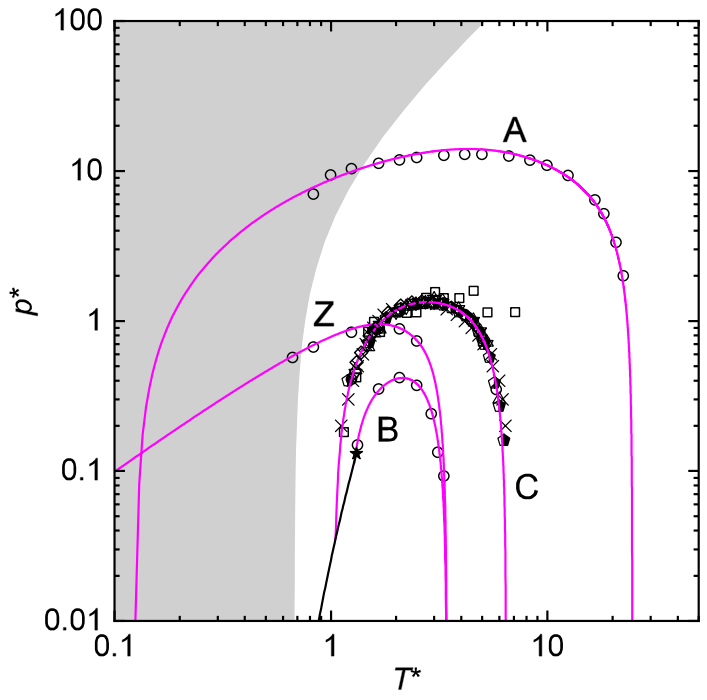

$\Delta \quad \nabla \quad$ Kioupis et al. [96]

- Deiters \& Neumaier [75]

- Heyes \& Llaguno [98]

- Vrabec et al. [97]

$\times \quad$ Colina \& Müller [99]

$\diamond \quad$ Yigzawe \& Sadus [100]

$\star \quad$ VLE \& crit. point Lafitte et al. [38] Lafitte et al. [38] 
Deiters and Neumaier [75] is used in the following for the evaluation of the LJ EOS. Figure 2 also shows the solid-fluid transition reported by Agrawal and Kofke [89].

It turns out that some of the simulation data of Deiters and Neumaier [75] for the Amagat and Charles curves probably lie beyond the freezing line. It is well known, however, that small simulation ensembles in cubic boxes with periodic boundary conditions tend to supercool. Deiters and Neumaier [75] used a moderate ensemble size of $N=1000$ particles, they always started their simulations from random configurations, and they monitored the simulations runs for signs of crystallization. One can therefore conclude that the reported simulation states beyond the freezing line represent supercool fluid states.

Figure 2 shows that the four characteristic curves computed from the LJ EOS of Lafitte et al. [38] satisfies all requirements postulated by Brown [70], i.e., starting points on the vapor pressure curve, limits at $p^{*} \rightarrow 0$, and intersection points, except the termination point of the Zeno and Amagat curve. Brown deduced from rational thermodynamic arguments that the Zeno and Amagat curve converge into each other in the zero-temperature and zero-pressure limit with infinite slope. However, it is interesting to note that the LJ EOS of Lafitte et al. [38] yields a crossing of the Zeno and Amagat curves at approximately the critical pressure. Furthermore, the Zeno curve of the LJ EOS of Lafitte et al. [38] does not exhibit an infinite slope in the zero-pressure limit as postulated by Brown [70].

Brown's [70] assumption for an infinite slope of the Zeno curve in the zero-pressure limit at low $T^{*}$ is probably incorrect. The compressibility factor on the Zeno curve is by definition $Z=1$, which yields $\ln p^{*}=\ln T^{*}+\ln \rho^{*}$. The last term converges approximately to a constant value at low $p^{*}$ for $T^{*} \rightarrow 0$. Hence, the Zeno curve has a constant slope of unity at $T^{*} \rightarrow 0$ in a double-logarithmic $p^{*}-T^{*}$ diagram - as predicted by the LJ EOS of Lafitte et al. [38] and others (see below). Nonetheless, for the LJ model that region lies in the solid region.

The characteristic curve computer experiment data of Deiters and Neumaier [75] are compared in Fig. 3 individually with the results obtained from the 20 investigated LJ EOS. The LJ EOS are ordered roughly according their types, i.e., starting from the physically motivated LJ EOS, to the semi-theoretical and fully empirical LJ EOS.

None except the LJ EOS of Lafitte et al. [38] satisfies all requirements for the characteristic curves and is in good quantitative agreement with available computer experiment data. The characteristic curves obtained from the LJ EOS of Ree [53] are in accordance with Brown's postulates, but show significant deviations from the computer experiment data. Most LJ EOS yield reasonable Zeno, Boyle, and Charles curves, but fail for the Amagat curve. In the case of inaccurate Zeno, Boyle, and Charles curves, they are mostly distorted at low temperatures. Several LJ EOS [41, 52, 56, 61, 67, 69] produce reasonable Amagat curves over a wide temperature range, but yield distortions in the vicinity of the solid-fluid equilibrium. There are also some LJ EOS that produce Amagat curves exhibiting minor oscillations at high pressures [28, 35, 51, 57, 60, 62-65], i.e., a wrong curvature.

A constant slope of unity for the Zeno curve at $T^{*} \rightarrow 0$ in the double-logarithmic $p^{*}-T^{*}$ diagram is also obtained from the LJ EOS of Refs. [35, 41, 46, 53, 56, 57 , 

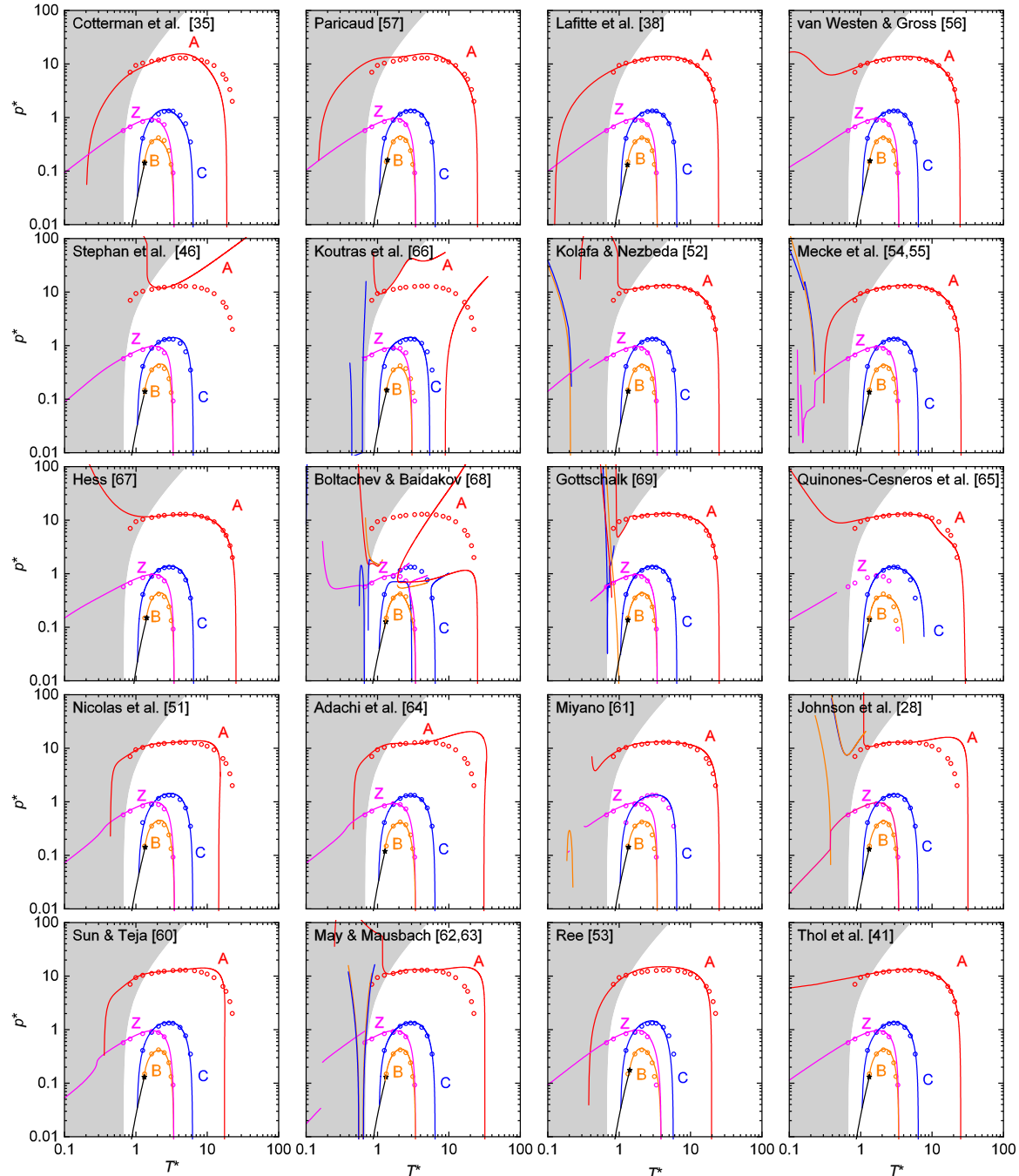

Fig. 3 Comparison of Brown's characteristic curves obtained from different LJ EOS (colored lines) with the molecular simulations results (symbols) of Deiters and Neumaier [75]. The black solid line and star indicate the VLE and critical point obtained from the respective LJ EOS. The gray-shaded region indicates the solid phase of the LJ potential as reported by Agrawal and Kofke [89]

67], cf. Fig. 3. This corroborates our argument concerning the original postulates of Brown [70].

The four characteristic curves studied here evidently represent challenges of different severity, i.e., the Charles curve is predicted qualitatively correct by most LJ EOS, while the Amagat curve is predicted qualitatively correct and in good agreement with reference data in the entire temperature range by merely one LJ EOS. 
There is a tendency among the four characteristic curves to be predicted qualitatively correct (Charles $\rightarrow$ Boyle $\rightarrow$ Zeno $\rightarrow$ Amagat).

Boshkova and Deiters [77] reported that many theory-based EOS fail to yield accurate Amagat curves due to simplifications in the modeling of the repulsive interactions. However, we find that the theory-based LJ EOS of Lafitte et al. [38], Cotterman et al. [35], and Paricaud [57] yield reasonable Amagat curves in a wide temperature range- the LJ EOS of Lafitte et al. [38] is even quantitatively in good agreement with computer experiment results.

The LJ EOS of Stephan et al. [46] based on the PC-SAFT monomer model yields qualitatively accurate Zeno, Boyle, and Charles curves, but a completely distorted Amagat curve. This type of behavior was also reported by Boshkova and Deiters [77] for the characteristic curves of the original PC-SAFT parametrization. They showed that this is a result of the simplified temperature-dependent diameter of the PC-SAFT approach which gives a poor description of the soft repulsion of the Lennard-Jones potential [77]. This is supported by the results from a recent work of our group [46], which showed that the LJ EOS of Stephan et al. (re-parametrized PCSAFT monomer term) yields large deviations at high temperatures and densities for most homogeneous state point properties, where the softness of the repulsive interactions becomes more important.

The LJ EOS of the MBWR type (Refs. [28, 51, 60-64]) have in common that they yield Zeno curves with a kink at low temperatures. Some of them also exhibit a distorted Boyle curve (LJ EOS of Refs. [28, 61-63]). All LJ EOS of the MBWR type yield distorted Amagat curves, but most yield accurate Charles curves. For some MBWR type LJ EOS [51, 60, 64], the Amagat curve has a positive curvature at high $T^{*}$.

The LJ EOS of Cotterman et al. [35], Paricaud [57], Thol et al. [41], Hess [67], and van Westen and Gross [56] produce qualitatively accurate Zeno, Boyle, and Charles curves, but distorted Amagat curves-especially at low temperatures. The LJ EOS of Stephan et al. [46] (re-parametrized PC-SAFT monomer), Koutras et al. [66], and Boltachev and Baidakov [68] yield erratic results for the Amagat curve. The LJ EOS of Cotterman et al. [35] and Paricaud [57] have a faint bump at high temperatures, i.e., wrong curvature. The LJ EOS of Boltachev and Baidakov [68] and Quiñones-Cisneros et al. [65] show a distorted shape for all four characteristic curves. For the LJ EOS of Quiñones-Cisneros et al. [65], the Zeno curve at moderate temperatures lies below $p^{*}=0.01$, i.e., out of the range of the depicted plot. The characteristic curves obtained from the LJ EOS of Gottschalk [69], Kolafa and Nezbeda [52], and Mecke et al. [54, 55] are in good agreement with the reference data in a wide temperature and pressure range of the fluid region, but all four curves yield unrealistic solutions at low temperatures (for the Amagat curve of Ref. [54, 55] at $\left.T^{*}<0.1\right)$. Overall, for most LJ EOS the identified deficiencies are found in the vicinity and beyond the solid-fluid equilibrium, i.e., the Zeno and Amagat curves, whereas a reasonable performance is often found at high temperatures. It should be noted that state points beyond the solid-fluid equilibrium can also be relevant for the modeling of fluid mixtures.

The characteristic curves obtained from the LJ EOS of Mecke et al. [54, 55] are in excellent agreement with the available computer experiment data, but show 
unphysical features in the low-temperature limit. Deiters and Neumaier [75] reported that the LJ EOS of Mecke et al. [54, 55] gives a realistic description of all characteristic curves, which is found differently in the present work. An additional (physically unrealistic $[70,77]$ ) branch is found for all four characteristic curves. Likewise, an unrealistic behavior is found for the Charles, Boyle, Zeno, and Amagat curve of the LJ EOS of Kolafa and Nezbeda [52] at low temperature.

The Amagat curve of the LJ EOS of Thol et al. [41] is distorted at lower temperatures-as also pointed out by Thol et al. [41] and Deiters and Neumaier [75]. However, we find a significantly different Amagat curve as reported by Thol et al. [41] for their LJ EOS. The Amagat curve computed from our implementation is in good agreement with the computer experiment results reported by Deiters and Neumaier [75] for most temperatures. We suspect a misprint in the publication of Thol et al. [41].

\subsection{Characteristic State Points}

The thermodynamic behavior of a pure substance contains multiple uniquely defined state points, of which the critical point is the most prominent one. Such state points can be favorably used to characterize the quality of EOS, since they comprise a condensed description of the thermodynamic behavior in a single state point. The critical point obtained from different LJ EOS in comparison to computer experiment data has been discussed in detail elsewhere [7, 46]. Here, characteristic state points related to the virial coefficients and Brown's characteristic curves are discussed. In particular, exact values for a given interaction potential can be obtained for some characteristic points from statistical mechanics.

The characteristic state points considered in the present work are schematically illustrated in Fig. 4; they are defined as:

- the state points of the four characteristic curves in the zero-density limit, labeled as $\mathcal{Z}\left(\rho^{*} \rightarrow 0\right), \mathcal{A}\left(\rho^{*} \rightarrow 0\right), \mathcal{B}\left(\rho^{*} \rightarrow 0\right)$, and $\mathcal{C}\left(\rho^{*} \rightarrow 0\right)$ (which can also be computed from the virial coefficients-see above),

- the zero crossing of the third virial coefficient $C^{*}\left(T^{*}\right)=0$, and the maximum of the third virial coefficient $\max \left(C^{*}\left(T^{*}\right)\right)$,

- the intersection of the Boyle and Charles curve with the vapor pressure curve labeled as VLE $\cap \mathcal{B}$ and VLE $\cap \mathcal{C}$,

- the intersection point of the Zeno and Charles curve labeled as $\mathcal{Z} \cap \mathcal{C}$,

- the maxima of the four characteristic curves in the $p^{*}-T^{*}$ plane labeled as $\max (\mathcal{A}), \max (\mathcal{B}), \max (\mathcal{C})$, and $\max (\mathcal{Z})$.

Exact values from numerical integration of the virial coefficients can be obtained for the temperature at $\mathcal{Z}\left(\rho^{*} \rightarrow 0\right), \mathcal{A}\left(\rho^{*} \rightarrow 0\right), \mathcal{B}\left(\rho^{*} \rightarrow 0\right)$ from the second virial coefficient (cf. Fig. 4-top and middle), the zero crossing of the third virial coefficient $C^{*}\left(T^{*}\right)=0$, and the maximum of the third virial coefficient $\max \left(C^{*}\left(T^{*}\right)\right)$ as indicated in Table 3 (cf. Fig. 4-bottom). These results are termed as 'results from the virial route' in the following. Those reported values in Table 3 were obtained in this 
Fig. 4 Scheme of the characteristic curves (top) and second and third virial coefficient (middle and bottom, respectively) for the illustration of the considered characteristic state points (red symbols), cf. Table 3
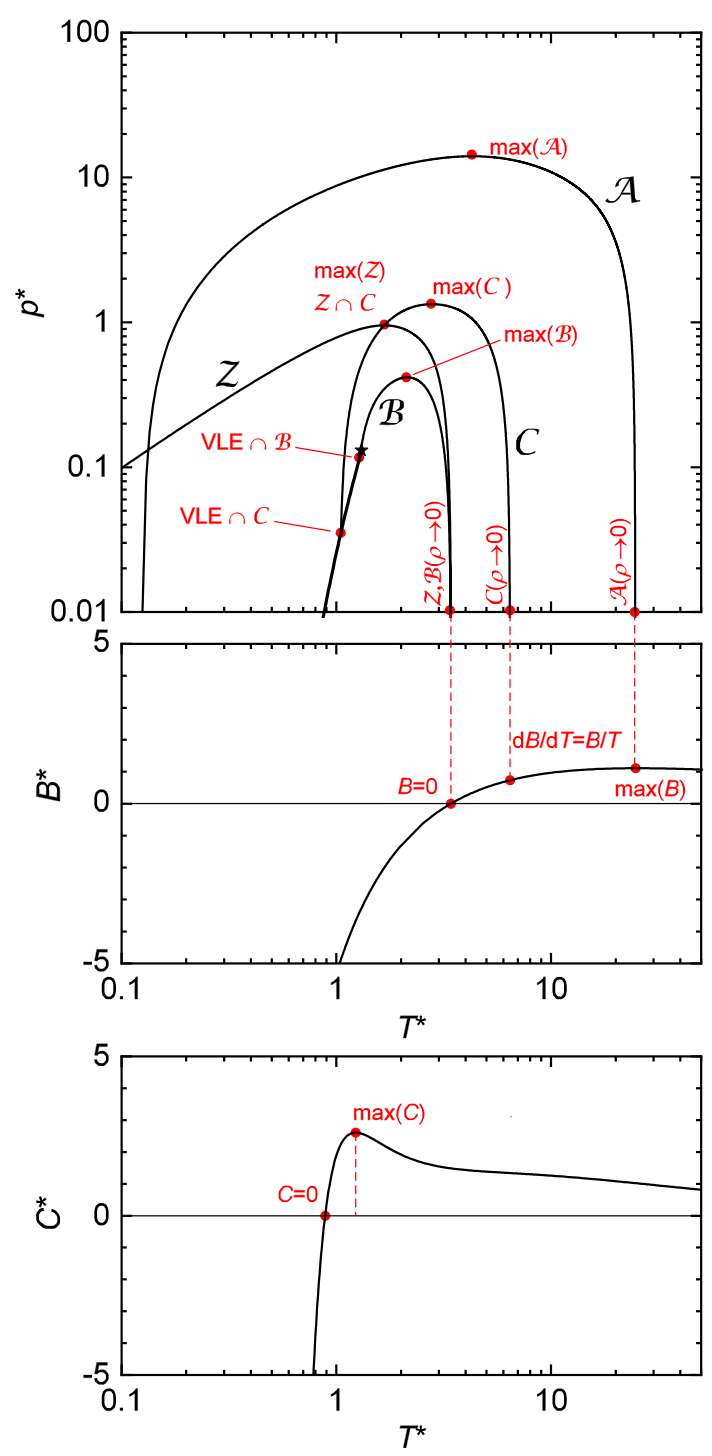

work and were-where available_-compared and found in excellent agreement with results from the literature [101].

Furthermore, the above-defined characteristic state points were computed for each of the considered LJ EOS. For the maxima and intersection points of the characteristic curves, an iterative solver was used to find the state point satisfying the respective conditions. For the intersection points of the characteristic curves with the phase envelope, both the VLE and the characteristic curves were iteratively computed by means of a given LJ EOS to find the intersection point. The 


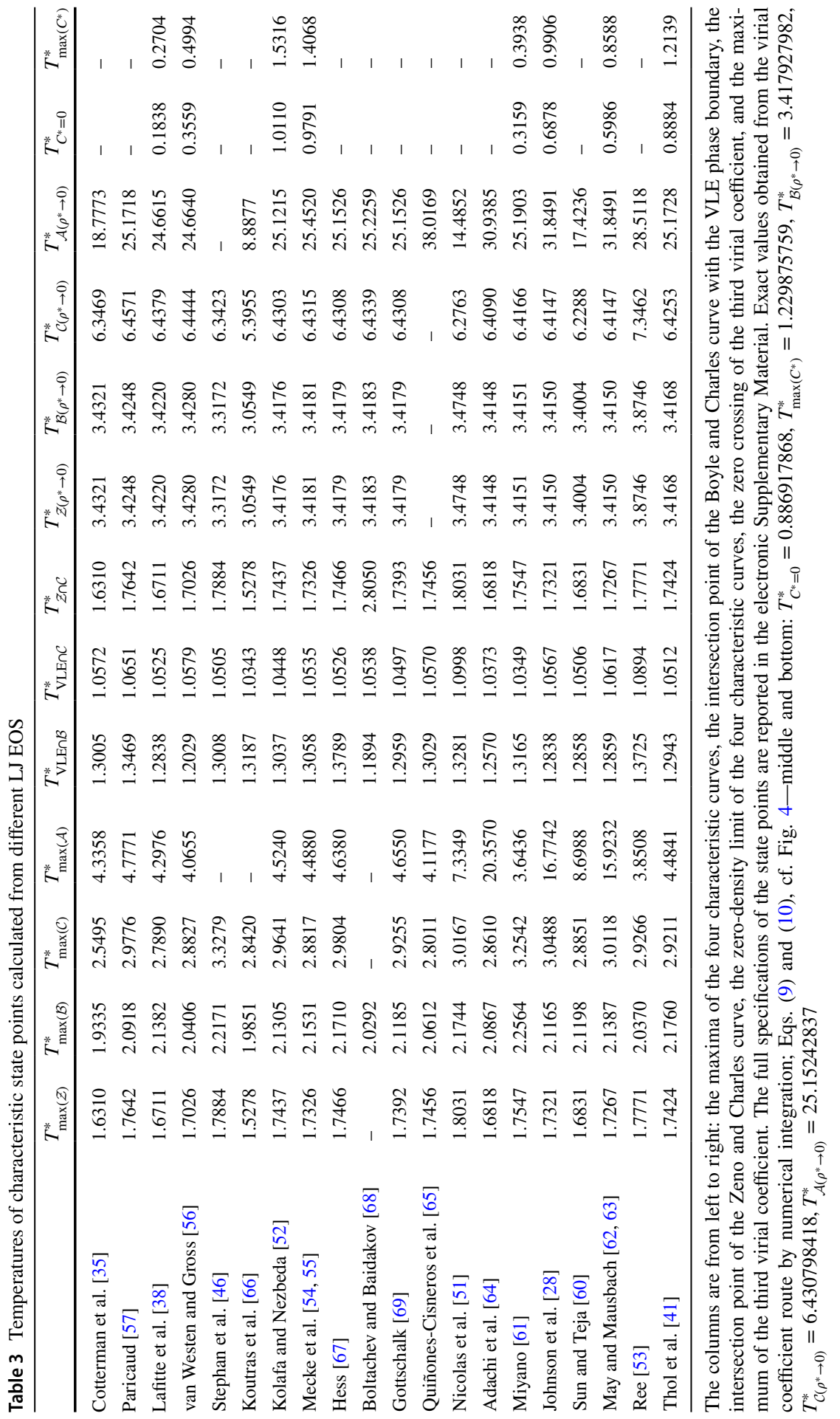


zero-density limit state points of the characteristic curves were computed directly from the definition of the respective curve at $\rho^{*} \rightarrow 0$. To validate the consistency of the LJ EOS implementations used in the present work, these state points were also computed by the LJ EOS from the corresponding definition from the second virial coefficient (see above) for comparison. The results obtained from the two thermodynamic definitions were found in all cases to be in perfect agreement.

Table 3 lists the temperatures of the characteristic state points. The temperature, pressure, and density of each state point are reported in the electronic Supplementary Material. The numeric values therein are reported with more decimal places than in Table 3. Blanks in Table 3 indicate cases where the shape of a characteristic curve or the third virial coefficient is distorted in a way that a maximum or crossing point could not be evaluated in a meaningful way.

The temperatures $T_{\mathcal{Z}\left(\rho^{*} \rightarrow 0\right)}^{*}, T_{\mathcal{B}\left(\rho^{*} \rightarrow 0\right)}^{*}, T_{\mathcal{C}\left(\rho^{*} \rightarrow 0\right)}^{*}$, and $T_{\mathcal{A}\left(\rho^{*} \rightarrow 0\right)}^{*}$ (zero-density limit state points of the characteristic curves) obtained from the LJ EOS can be compared with exact results computed by numerical integration via the virial coefficient route, cf. Fig. 4-middle and Table 3. Excellent agreement is found for many LJ EOS except the LJ EOS of Refs. [46, 51, 53, 56, 60, 65, 66] which yield significantly deviating $T_{\mathcal{Z}\left(\rho^{*} \rightarrow 0\right)}^{*}$ and $T_{\mathcal{B}\left(\rho^{*} \rightarrow 0\right)}^{*} ;$ the LJ EOS of Refs. [28, 35, 46, 51, 53, 60, 62-66] yield significantly deviating $T_{\mathcal{A}\left(\rho^{*} \rightarrow 0\right)}^{*}$ (or even no solution).

The zero-density limits $T_{\mathcal{Z}\left(\rho^{*} \rightarrow 0\right)}^{*}, T_{\mathcal{B}\left(\rho^{*} \rightarrow 0\right)}^{*}, T_{\mathcal{C}\left(\rho^{*} \rightarrow 0\right)}^{*}$, and $T_{\mathcal{A}\left(\rho^{*} \rightarrow 0\right)}^{*}$ obtained from the LJ EOS of Gottschalk [69] and Hess [67] agree with the exact values within the computer precision employed for the calculations since those are integrated in the respective equation. For the LJ EOS of Paricaud [57] small deviations for the $T_{\mathcal{Z}\left(\rho^{*} \rightarrow 0\right)}^{*}, T_{\mathcal{B}\left(\rho^{*} \rightarrow 0\right)}^{*}, T_{\mathcal{C}\left(\rho^{*} \rightarrow 0\right)}^{*}$, and $T_{\mathcal{A}\left(\rho^{*} \rightarrow 0\right)}^{*}$ in comparison to the exact data are found, which is in line with the small deviations observed for the second virial coefficient itself. Also the LJ EOS of Kolafa and Nezbeda [52] has a second virial coefficient term and therefore gives an excellent description of the zero-density limits of the characteristic curves. Also the empirical LJ EOS of Thol et al. [41] yields accurate results for $T_{\mathcal{Z}\left(\rho^{*} \rightarrow 0\right)}^{*}, T_{\mathcal{B}\left(\rho^{*} \rightarrow 0\right)}^{*}, T_{\mathcal{C}\left(\rho^{*} \rightarrow 0\right)}^{*}$, and $T_{\mathcal{A}\left(\rho^{*} \rightarrow 0\right)}^{*}$ (deviations below $2 \%$ ).

Exact values were also obtained in the present work for the temperature of the zero crossing and maximum of the third virial coefficient $T_{C^{*}=0}^{*}$ and $T_{\max \left(C^{*}\right)}^{*}$ from numerical integration, cf. Table 3. Only results from the LJ EOS of Refs. [41, 52, $54,55]$ are found to be in good agreement with exact values for $T_{C^{*}=0}^{*}$ and $T_{\max \left(C^{*}\right)}^{*}$; reasonable agreement is found for the LJ EOS of Refs. [28, 62, 63]. The best results for $T_{C^{*}=0}^{*}$ and $T_{\max \left(C^{*}\right)}^{*}$ are obtained from the LJ EOS of Thol et al. [41].

The temperature of the Zeno curve maximum $T_{\max (\mathcal{Z})}^{*}$ obtained from the 20 investigated LJ EOS are in good agreement. They lie in the range $T_{\max (\mathcal{Z})}^{*}=1.74 \pm 0.06$. The temperature of the Boyle curve maximum obtained from the $20 \mathrm{LJ}$ EOS scatters slightly more in the range $T_{\max (\mathcal{B})}^{*}=2.12 \pm 0.09$. Only the LJ EOS of Koutras et al. [66] yields a significantly lower $T_{\max (\mathcal{Z})}^{*}$ and $T_{\max (\mathcal{B})}^{*}$ compared to all other investigated LJ EOS. The scattering is significantly larger for the temperature of the Charles curve maximum obtained from the different LJ EOS as $T_{\max (\mathcal{C})}^{*}=2.92 \pm 0.5$. For the temperature of the Amagat curve maximum, 11 LJ EOS scatter around $T_{\max (\mathcal{A})}^{*}=4.5 \pm 1.5$. The LJ EOS of Refs. [28, 35, 60, 62-64, 68] show significantly 
shifted $T_{\max (\mathcal{A})}^{*}$ which is due to the distorted Amagat curves produced by these LJ EOS.

The differences in the intersection points of the Charles and Boyle curves with the VLE are dominated by differences in the VLE obtained from the different LJ EOS - especially close to the critical point. The temperature of the Zeno and Charles curves intersection point (corresponding to $\max (\mathcal{Z})$ ) obtained from the considered LJ EOS agrees within $T_{\mathcal{Z} \cap \mathcal{C}}^{*}=1.74 \pm 0.06$, excluding the LJ EOS of Ref. [68].

Sadus [101] recently reported values for the Boyle temperature and the maximum of the second virial coefficient computed from the LJ EOS of Koutras et al. [66], which significantly deviate from the values obtained from our implementation for that LJ EOS. However, we compared $B^{*}\left(T^{*}\right)$ obtained from our implementation with results originally reported by Koutras et al. [66] and found excellent agreement.

\section{Conclusions}

The present work revisits Brown's characteristic curves and virial coefficients of the Lennard-Jones fluid. They were computed from a large number of LJ EOS and from rigorous statistical mechanics (where accessible). For most LJ EOS, these properties have not been examined yet-especially the physically motivated ones.

The second virial coefficient is predicted qualitatively correctly by all but one LJ EOS. However, significant quantitative deviations are observed for most considered LJ EOS. For the third virial coefficient, only few LJ EOS produce qualitatively correct results.

Brown's characteristic curves [70] predicted from the different LJ EOS were compared with computer experiment data of Deiters and Neumaier [75] and with exact values in the ideal gas limit. The physically motivated LJ EOS are found to give an overall better description of the characteristic curves-especially at low temperature and high pressure. Most LJ EOS produce distorted Amagat curves. Only the LJ EOS of Lafitte et al. [38] yields realistic results for all characteristic curves in the entire temperature range that are also in good quantitative agreement with available computer experiment data. The LJ EOS of Ree [53] yields realistic descriptions for the characteristic curves, but significant deviation to computer experiment data. Nevertheless, in several cases, a reasonable performance is found in a wide temperature range, e.g., the LJ EOS of Refs. [35, 41, 56, 57, 67, 69].

We showed that Brown's assumption that the Amagat and Zeno curves should converge with an infinite slope in the zero-pressure limit at low temperatures (in the double-logarithmic pressure-temperature diagram) is probably inaccurate. The Zeno curve exhibits a limiting slope of unity. Hence, the required intersection point of the Amagat and Zeno curves is not found in the zero-pressure limit.

Brown's characteristic curves are found to be sensitive properties, in a sense that they clearly reveal unphysical behavior of an EOS, which holds in particular for the Amagat and Zeno curve. The Boyle and Charles curve are found to be predicted accurately by most LJ EOS and are therefore less sensitive indicators. Nevertheless, the application of the characteristic curves to investigate 'the extrapolation behavior 
of EOS' [73] should be carried out with caution. For example, the characteristic curves from the LJ EOS of Mecke et al. [54, 55] and Lafitte et al. [38] are in good agreement with corresponding computer experiment data in a wide temperature range, but both LJ EOS exhibit large deviations from pressure and internal energy reference data at extreme temperature and density (beyond the Amagat curve), cf. Ref. [46]. Vice versa, the LJ EOS of Kolafa and Nezbeda [52] and Thol et al. [41] exhibit distorted Amagat curves at low temperatures, but both LJ EOS give an overall accurate and fairly precise description of pressure and internal energy reference data also at extreme conditions [46]. Hence, for these LJ EOS the findings for the performance on the characteristic curves could not be transferred to conditions significantly above the pressure and temperature range of the characteristic curves. Instead, it is emphasized that the characteristic curves are a necessary requirement for an EOS to be accurate in the entire temperature and pressure range, but not a sufficient one.

Acknowledgements The authors gratefully acknowledge funding of the present work by the ERC Advanced Grant ENRICO (grant agreement No. 694807) and by the Deutsche Forschungsgemeinschaft—DFG (grant IRTG 2057-252408385 "Physical Modelling for Virtual Manufacturing Systems and Processes"). The authors thank Hans Hasse for fruitful discussions and Jens Staubach for supporting the calculation work. The present research was conducted under the auspices of the Boltzmann-Zuse Society of Computational Molecular Engineering (BZS).

Funding Open Access funding provided by Projekt DEAL.

\section{Compliance with Ethical Standards}

Conflict of interest The authors declare that they have no conflict of interest.

Open Access This article is licensed under a Creative Commons Attribution 4.0 International License, which permits use, sharing, adaptation, distribution and reproduction in any medium or format, as long as you give appropriate credit to the original author(s) and the source, provide a link to the Creative Commons licence, and indicate if changes were made. The images or other third party material in this article are included in the article's Creative Commons licence, unless indicated otherwise in a credit line to the material. If material is not included in the article's Creative Commons licence and your intended use is not permitted by statutory regulation or exceeds the permitted use, you will need to obtain permission directly from the copyright holder. To view a copy of this licence, visit http://creativecommons.org/licen ses/by/4.0/.

\section{References}

1. J. Jones, On the determination of molecular fields. I. from the variation of the viscosity of a gas with temperature. Proc. R. Soc. Lond. Ser. A 106(738), 441-462 (1924a). https://doi.org/10.1098/ rspa.1924.0081

2. J. Jones, On the determination of molecular fields. II. from the equation of state of a gas. Proc. R. Soc. Lond. Ser. A 106(738), 463-477 (1924b). https://doi.org/10.1098/rspa.1924.0082

3. N. Metropolis, A.W. Rosenbluth, M.N. Rosenbluth, A.H. Teller, E. Teller, Equation of state calculations by fast computing machines. J. Chem. Phys. 21(6), 1087-1092 (1953). https://doi. org/10.1063/1.1699114 
4. M.N. Rosenbluth, A.W. Rosenbluth, Further results on Monte Carlo equations of state. J. Chem. Phys. 22(5), 881-884 (1954). https://doi.org/10.1063/1.1740207

5. W.W. Wood, F.R. Parker, Monte Carlo equation of state of molecules interacting with the LennardJones potential. I. A supercritical isotherm at about twice the critical temperature. J. Chem. Phys. 27(3), 720-733 (1957). https://doi.org/10.1063/1.1743822

6. B.J. Alder, T.E. Wainwright, Studies in molecular dynamics. I. general method. J. Chem. Phys. 31(2), 459-466 (1959). https://doi.org/10.1063/1.1730376

7. S. Stephan, M. Thol, J. Vrabec, H. Hasse, Thermophysical properties of the Lennard-Jones fluid: database and data assessment. J. Chem. Inf. Model. 59(10), 4248-4265 (2019). https://doi. org/10.1021/acs.jcim.9b00620

8. M.M. Telo da Gama, R. Evans, The structure and surface tension of the liquid-vapour interface near the upper critical end point of a binary mixture of Lennard-Jones fluids I. the two phase region. Mol. Phys. 48(2), 229-250 (1983). https://doi.org/10.1080/00268978300100181

9. J.D. Honeycutt, H.C. Andersen, Molecular dynamics study of melting and freezing of small Lennard-Jones clusters. J. Phys. Chem. 91(19), 4950-4963 (1987). https://doi.org/10.1021/j100303a01 4

10. S. Stephan, H. Hasse, Molecular interactions at vapor-liquid interfaces: binary mixtures of simple fluids. Phys. Rev. E 101, 012802 (2020). https://doi.org/10.1103/PhysRevE.101.012802

11. S. Stephan, J. Liu, K. Langenbach, W.G. Chapman, H. Hasse, Vapor-liquid interface of the Lennard-Jones truncated and shifted fluid: comparison of molecular simulation, density gradient theory, and density functional theory. J. Phys. Chem. C 122(43), 24705-24715 (2018). https://doi. org/10.1021/acs.jpcc.8b06332

12. A. Köster, P. Mausbach, J. Vrabec, Premelting, solid-fluid equilibria, and thermodynamic properties in the high density region based on the Lennard-Jones potential. J. Chem. Phys. 147(14), 144502 (2017). https://doi.org/10.1063/1.4990667

13. S.M. Thomson, K.E. Gubbins, J.P. Walton, R.A. Chantry, J.S. Rowlinson, A molecular dynamics study of liquid drops. J. Chem. Phys. 81, 530 (1984)

14. P.R. ten Wolde, D. Frenkel, Computer simulation study of gas-liquid nucleation in a Lennard-Jones system. J. Chem. Phys. 109(22), 9901-9918 (1998). https://doi.org/10.1063/1.477658

15. M. Mecke, J. Winkelmann, J. Fischer, Molecular dynamics simulation of the liquid-vapor interface: the Lennard-Jones fluid. J. Chem. Phys. 107(21), 9264-9270 (1997). https://doi. org/10.1063/1.475217

16. A. Mejía, J.C. Pàmies, D. Duque, H. Segura, L.F. Vega, Phase and interface behaviors in type-I and type-V Lennard-Jones mixtures: Theory and simulations. J. Chem. Phys. 123(3), 034505 (2005). https://doi.org/10.1063/1.1955529

17. M. Horsch, J. Vrabec, H. Hasse, Modification of the classical nucleation theory based on molecular simulation data for surface tension, critical nucleus size, and nucleation rate. Phys. Rev. E 78(1), 011603 (2008). https://doi.org/10.1103/PhysRevE.78.011603

18. S. Stephan, M. Dyga, H. Urbassek, H. Hasse, The influence of lubrication and the solid-fluid interaction on thermodynamic properties in a nanoscopic scratching process. Langmuir 35(51), 1694816960 (2019). https://doi.org/10.1021/acs.langmuir.9b01033

19. S. Stephan, M.P. Lautenschlaeger, I.A. Alhafez, M.T. Horsch, H.M. Urbassek, H. Hasse, Molecular dynamics simulation study of mechanical effects of lubrication on a nanoscale contact process. Tribol. Lett. 66(4), 126 (2018). https://doi.org/10.1007/s11249-018-1076-0

20. B.L. Eggimann, A.J. Sunnarborg, H.D. Stern, A.P. Bliss, J.I. Siepmann, An online parameter and property database for the TraPPE force field. Mol. Simul. 40(1-3), 101-105 (2014). https://doi. org/10.1080/08927022.2013.842994

21. J.L.F. Abascal, C. Vega, A general purpose model for the condensed phases of water: TIP4P/2005. J. Chem. Phys. 123(23), 234505 (2005). https://doi.org/10.1063/1.2121687

22. S. Stephan, M. Horsch, J. Vrabec, H. Hasse, MolMod-an open access database of force fields for molecular simulations of fluids. Mol. Simul. 45(10), 806-814 (2019). https://doi. org/10.1080/08927022.2019.1601191

23. W. Shi, J. Johnson, Histogram reweighting and finite-size scaling study of the Lennard-Jones fluids. Fluid Phase Equilibria 187, 171-191 (2001). https://doi.org/10.1016/S0378-3812(01)00534-9

24. D.O. Dunikov, S.P. Malyshenko, V.V. Zhakhovskii, Corresponding states law and molecular dynamics simulations of the Lennard-Jones fluid. J. Chem. Phys. 115(14), 6623-6631 (2001). https ://doi.org/10.1063/1.1396674 
25. J. Powles, The liquid-vapour coexistence line for Lennard-Jones-type fluids. Physica A 126(1), 289-299 (1984). https://doi.org/10.1016/0378-4371(84)90156-0

26. B. Smit, Phase diagrams of Lennard-Jones fluids. J. Chem. Phys. 96(11), 8639-8640 (1992). https ://doi.org/10.1063/1.462271

27. V. Baidakov, G. Chernykh, S. Protsenko, Effect of the cut-off radius of the intermolecular potential on phase equilibrium and surface tension in Lennard-Jones systems. Chem. Phys. Lett. 321(3), 315-320 (2000). https://doi.org/10.1016/S0009-2614(00)00217-7

28. J.K. Johnson, J.A. Zollweg, K.E. Gubbins, The Lennard-Jones equation of state revisited. Mol. Phys. 78(3), 591 (1993). https://doi.org/10.1080/00268979300100411

29. K.R.S. Shaul, A.J. Schultz, D.A. Kofke, The effect of truncation and shift on virial coefficients of Lennard-Jones potentials. Collect. Czechoslovak Chem. Commun. 75(4), 447-462 (2010). https:// doi.org/10.1135/ccce2009113

30. V.K. Shen, R.D. Mountain, J.R. Errington, Comparative study of the effect of tail corrections on surface tension determined by molecular simulation. J. Phys. Chem. B 111(22), 6198-6207 (2007). https://doi.org/10.1021/jp070374f

31. F.J. Martinez-Ruiz, F.J. Blas, B. Mendiboure, A.I. Moreno-Ventas Bravo, Effect of dispersive longrange corrections to the pressure tensor: The vapour-liquid interfacial properties of the LennardJones system revisited. J. Chem. Phys. 141(18), 184701 (2014). https://doi.org/10.1063/1.4900773

32. S. Stephan, H. Hasse, Influence of dispersive long-range interactions on properties of vapour-liquid equilibria and interfaces of binary Lennard-Jones mixtures. Mol. Phys. 118(9-10), e1699185 (2020). https://doi.org/10.1080/00268976.2019.1699185

33. M.P. Allen, D.J. Tildesley, Computer simulation of liquids (Oxford University Press, Oxford, 1989)

34. W.G. Chapman, G. Jackson, K.E. Gubbins, M. Radosz, New reference equation of state for associating liquids. Ind. Eng. Chem. Res. 29, 1709 (1990)

35. R.L. Cotterman, B.J. Schwarz, J.M. Prausnitz, Molecular thermodynamics for fluids at low and high densities. Part I: Pure fluids containing small or large molecules. AIChE J. 32(11), 1787-1798 (1986). https://doi.org/10.1002/aic.690321104

36. F.J. Blas, L.F. Vega, Thermodynamic behaviour of homonuclear and heteronuclear Lennard-Jones chains with association sites from simulation and theory. Mol. Phys. 92, 135-150 (1997). https:// doi.org/10.1080/002689797170707

37. F.J. Blas, L.F. Vega, Prediction of binary and ternary diagrams using the statistical associating fluid theory (SAFT) equation of state. Ind. Eng. Chem. Res. 37(2), 660-674 (1998). https://doi. org/10.1021/ie970449+

38. T. Lafitte, A. Apostolakou, C. Avendano, A. Galindo, C.S. Adjiman, E.A. Müller, G. Jackson, Accurate statistical associating fluid theory for chain molecules formed from Mie segments. J. Chem. Phys. 139(15), 154504 (2013). https://doi.org/10.1063/1.4819786

39. U.K. Deiters, K.M. De Reuck, Guidelines for publication of equations of state-I. pure fluids. Chem. Eng. J. 69(1), 69-81 (1998). https://doi.org/10.1016/S1385-8947(97)00070-3

40. M. Thol, G. Rutkai, R. Span, J. Vrabec, R. Lustig, Equation of state for the Lennard-Jones truncated and shifted model fluid. Int. J. Thermophys. 36(1), 25 (2015). https://doi.org/10.1007/s1076 5-014-1764-4

41. M. Thol, G. Rutkai, A. Köster, R. Lustig, R. Span, J. Vrabec, Equation of state for the LennardJones fluid. J. Phys. Chem. Ref. Data 45(2), 023101 (2016). https://doi.org/10.1063/1.4945000

42. M. Heier, S. Stephan, J. Liu, W.G. Chapman, H. Hasse, K. Langenbach, Equation of state for the Lennard-Jones truncated and shifted fluid with a cut-off radius of 2.5 based on perturbation theory and its applications to interfacial thermodynamics. Mol. Phys. 116(15), 2083-2094 (2018). https:// doi.org/10.1080/00268976.2018.1447153

43. O. Pohl, Correspondence - evaluation of an improved volume translation for the prediction of hydrocarbon volumetric properties. Fluid Phase Equilibria 163(1), 157-159 (1999). https://doi. org/10.1016/S0378-3812(99)00199-5

44. L.V. Yelash, T. Kraska, Volume-translated equations of state: empirical approach and physical relevance. AIChE J. 49(6), 1569-1579 (2003). https://doi.org/10.1002/aic.690490620

45. J. Ahlers, J. Gmehling, Development of an universal group contribution equation of state: I. prediction of liquid densities for pure compounds with a volume translated Peng-Robinson equation of state. Fluid Phase Equilibria 191(1), 177-188 (2001). https://doi.org/10.1016/S0378 $-3812(01) 00626-4$

46. S. Stephan, J. Staubach, H. Hasse, Review and comparison of equations of state for the LennardJones fluid. Fluid Phase Equilibria 523, 112772 (2020). https://doi.org/10.1016/j.fluid.2020.112772 
47. W.G. Chapman, G. Jackson, K.E. Gubbins, Phase equilibira of associating fluids. Chain molecules with multiple bonding sites. Mol. Phys. 65, 1057-1079 (1988)

48. W.G. Chapman, K.E. Gubbins, G. Jackson, M. Radosz, SAFT: equation-of-state solution model for associating fluids. Fluid Phase Equilibria 52, 31-38 (1989). https://doi.org/10.1016/03783812(89)80308-5

49. V. Papaioannou, T. Lafitte, C. Avendano, C.S. Adjiman, G. Jackson, E.A. Müller, A. Galindo, Group contribution methodology based on the statistical associating fluid theory for heteronuclear molecules formed from Mie segments. J. Chem. Phys. 140(5), 054107 (2014). https://doi. org/10.1063/1.4851455

50. J. Gross, G. Sadowski, Perturbed-Chain SAFT: an equation of state based on a perturbation theory for chain molecules. Ind. Eng. Chem. Res. 40(4), 1244 (2001). https://doi.org/10.1021/ie0003887

51. J.J. Nicolas, K.E. Gubbins, W.B. Streett, D.J. Tildesley, Equation of state for the Lennard-Jones fluid. Mol. Phys. 37(5), 1429 (1979). https://doi.org/10.1080/00268977900101051

52. J. Kolafa, I. Nezbeda, The Lennard-Jones fluid: an accurate analytic and theoretically-based equation of state. Fluid Phase Equilibria 100, 1-34 (1994). https://doi.org/10.1016/0378-3812(94)80001 $-4$

53. F.H. Ree, Analytic representation of thermodynamic data for the Lennard-Jones fluid. J. Chem. Phys. 73(10), 5401-5403 (1980). https://doi.org/10.1063/1.439940

54. M. Mecke, A. Müller, J. Winkelmann, J. Vrabec, J. Fischer, R. Span, W. Wagner, An accurate van der Waals-type equation of state for the Lennard-Jones fluid. Int. J. Thermophys. 17(2), 391-404 (1996). https://doi.org/10.1007/BF01443399

55. M. Mecke, A. Müller, J. Winkelmann, J. Vrabec, J. Fischer, R. Span, W. Wagner, Erratum —an accurate van der Waals-type equation of state for the Lennard-Jones fluid. Int. J. Thermophys. 19(5), 14931498 (1998). https://doi.org/10.1023/A:1021995905315

56. T. van Westen, J. Gross, A critical evaluation of perturbation theories by Monte Carlo simulation of the first four perturbation terms in a Helmholtz energy expansion for the Lennard-Jones fluid. J. Chem. Phys. 147(1), 014503 (2017). https://doi.org/10.1063/1.4991008

57. P. Paricaud, A general perturbation approach for equation of state development: applications to simple fluids, ab initio potentials, and fullerenes. J. Chem. Phys. 124(15), 154505 (2006). https://doi. org/10.1063/1.2181979

58. J.A. Barker, D. Henderson, Theories of liquids. Ann. Rev. Phys. Chem. 23(1), 439-484 (1972). https:// doi.org/10.1146/annurev.pc.23.100172.002255

59. J.A. Barker, D. Henderson, Perturbation theory and equation of state for fluids. II. a successful theory of liquids. J. Chem. Phys. 47(11), 4714 (1967). https://doi.org/10.1063/1.1701689

60. T. Sun, A.S. Teja, An equation of state for real fluids based on the Lennard-Jones potential. J. Phys. Chem. 100(43), 17365-17372 (1996). https://doi.org/10.1021/jp9620476

61. Y. Miyano, An equation of state for Lennard-Jones pure fluids applicable over a very wide temperature range. Fluid Phase Equilibria 85, 71-80 (1993). https://doi.org/10.1016/0378-3812(93)80005-8

62. H.O. May, P. Mausbach, Riemannian geometry study of vapor-liquid phase equilibria and supercritical behavior of the Lennard-Jones fluid. Phys. Rev. E 85, 031201 (2012a). https://doi.org/10.1103/PhysR evE.85.031201

63. H.O. May, P. Mausbach, Erratum: Riemannian geometry study of vapor-liquid phase equilibria and supercritical behavior of the Lennard-Jones fluid [Phys. Rev. E 85, 031201 (2012)]. Phys. Rev. E 86, 059905 (2012b). https://doi.org/10.1103/PhysRevE.86.059905

64. Y. Adachi, I. Fijihara, M. Takamiya, K. Nakanishi, Generalized equation of state for Lennard-Jones fluids-I. pure fluids and simple mixtures. Fluid Phase Equilibria 39(1), 1-38 (1988). https://doi. org/10.1016/0378-3812(88)80001-3

65. S.E. Quiñones-Cisneros, U.K. Deiters, R.E. Rozas, T. Kraska, New model for the correlation of the surface tension based on friction theory. J. Phys. Chem. B 113(11), 3504-3511 (2009). https://doi. org/10.1021/jp8073255

66. N. Koutras, V. Harismiadis, D. Tassios, A simple equation of state for the Lennard-Jones fluid: a new reference term for equations of state and perturbation theories. Fluid Phase Equilibria 77, 13-38 (1992). https://doi.org/10.1016/0378-3812(92)85097-R

67. S. Hess, Augmented van der Waals equation of state for the Lennard-Jones fluid. Physica A 267(1), 58-70 (1999). https://doi.org/10.1016/S0378-4371(98)00670-0

68. G.S. Boltachev, V.G. Baidakov, Equation of state for Lennard-Jones fluid. High Temp. 41(2), 270-272 (2003). https://doi.org/10.1023/A:1023394122000 
69. M. Gottschalk, An EOS for the Lennard-Jones fluid: a virial expansion approach. AIP Adv. 9(12), 125206 (2019). https://doi.org/10.1063/1.5119761

70. E.H. Brown, On the thermodynamic properties of fluids. Bulletin de l'Institut International du Froid Annexe 1960-1, 169-178 (1960)

71. H. Hasse, J. Lenhard, Boon and bane: on the role of adjustable parameters in simulation models, in Mathematics as a Tool: Tracing New Roles of Mathematics in the Sciences, ed. by J. Lenhard, M. Carrier (Springer, Berlin, 2017), pp. 93-115. https://doi.org/10.1007/978-3-319-54469-4_6

72. M. Schappals, A. Mecklenfeld, L. Kröger, V. Botan, A. Köster, S. Stephan, E.J. Garcia, G. Rutkai, G. Raabe, P. Klein, K. Leonhard, C.W. Glass, J. Lenhard, J. Vrabec, H. Hasse, Round robin study: molecular simulation of thermodynamic properties from models with internal degrees of freedom. J. Chem. Theory Comput. 13(9), 4270-4280 (2017). https://doi.org/10.1021/acs.jctc.7b00489

73. R. Span, W. Wagner, On the extrapolation behavior of empirical equations of state. Int. J. Thermophys. 18(6), 1415-1443 (1997). https://doi.org/10.1007/BF02575343

74. A. Neumaier, U.K. Deiters, The characteristic curves of water. Int. J. Thermophys. 37(9), 96 (2016). https ://doi.org/10.1007/s10765-016-2098-1

75. U.K. Deiters, A. Neumaier, Computer simulation of the characteristic curves of pure fluids. J. Chem. Eng. Data 61(8), 2720-2728 (2016). https://doi.org/10.1021/acs.jced.6b00133

76. U.K. Deiters, K.M. De Reuck, Guidelines for publication of equations of state-I. Pure fluids. Pure Appl. Chem. 69(6), 1237-1250 (1998). https://doi.org/10.1351/pac199769061237

77. O.L. Boshkova, U.K. Deiters, Soft repulsion and the behavior of equations of state at high pressures. Int. J. Thermophys. 31(2), 227-252 (2010). https://doi.org/10.1007/s10765-010-0727-7

78. E. Mason, T. Spurling, The Virial Equation of State (Pergamon Press, Oxford, 1969)

79. T.L. Hill, An Introduction to Statistical Thermodynamics (Dover Publications, New York, 1986)

80. A. Hutem, S. Boonchui, Numerical evaluation of second and third virial coefficients of some inert gases via classical cluster expansion. J. Math. Chem. 50(5), 1262-1276 (2012). https://doi.org/10.1007/ s10910-011-9966-5

81. T.L. Hill, Statistical Mechanics (McGraw-Hill Book, New York, 1956)

82. J.O. Hirschfelder, C.F. Curtiss, R.B. Bird, Molecular Theory of Gases and Liquids (Wiley, New York, 1954)

83. J.A. Barker, P.J. Leonard, A. Pompe, Fifth virial coefficients. J. Chem. Phys. 44(11), 4206-4211 (1966). https://doi.org/10.1063/1.1726606

84. R.J. Wheatley, Calculation of high-order virial coefficients with applications to hard and soft spheres. Phys. Rev. Lett. 110, 200601 (2013). https://doi.org/10.1103/PhysRevLett.110.200601

85. A. Ahmed, R.J. Sadus, Solid-liquid equilibria and triple points of n-6 Lennard-Jones fluids. J. Chem. Phys. 131(17), 174504 (2009). https://doi.org/10.1063/1.3253686

86. A. Ahmed, R.J. Sadus, Erratum: Solid-liquid equilibria and triple points of n-6 Lennard-Jones fluids [J. Chem. Phys. 131, 174504 (2009)]. J. Chem. Phys. 133(22), 229902 (2010). https://doi. org/10.1063/1.3512996

87. E.A. Mastny, J.J. de Pablo, Melting line of the Lennard-Jones system, infinite size, and full potential. J. Chem. Phys. 127(10), 104504 (2007). https://doi.org/10.1063/1.2753149

88. A.J. Schultz, D.A. Kofke, Comprehensive high-precision high-accuracy equation of state and coexistence properties for classical Lennard-Jones crystals and low-temperature fluid phases. J. Chem. Phys. 149(20), 204508 (2018). https://doi.org/10.1063/1.5053714

89. R. Agrawal, D.A. Kofke, Thermodynamic and structural properties of model systems at solid-fluid coexistence. Mol. Phys. 85(1), 43-59 (1995). https://doi.org/10.1080/00268979500100921

90. A. Ladd, L. Woodcock, Interfacial and co-existence properties of the Lennard-Jones system at the triple point. Mol. Phys. 36(2), 611-619 (1978). https://doi.org/10.1080/00268977800101791

91. J.P. Hansen, L. Verlet, Phase transitions of the Lennard-Jones system. Phys. Rev. 184(1), 151-161 (1969). https://doi.org/10.1103/PhysRev.184.151

92. J.M.G. Sousa, A.L. Ferreira, M.A. Barroso, Determination of the solid-fluid coexistence of the n-6 Lennard-Jones system from free energy calculations. J. Chem. Phys. 136(17), 174502 (2012). https://doi. org $/ 10.1063 / 1.4707746$

93. R.B. Bird, E.L. Spotz, J.O. Hirschfelder, The third virial coefficient for non-polar gases. J. Chem. Phys. 18(10), 1395-1402 (1950). https://doi.org/10.1063/1.1747484

94. F. Castro-Marcano, C.G. Olivera-Fuentes, C.M. Colina, Joule-Thomson inversion curves and third virial coefficients for pure fluids from molecular-based models. Ind. Eng. Chem. Res. 47(22), 8894-8905 (2008). https://doi.org/10.1021/ie800651q 
95. J. Gross, G. Sadowski, Application of the perturbed-chain SAFT equation of state to associating systems. Ind. Eng. Chem. Res. 41(22), 5510-5515 (2002). https://doi.org/10.1021/ie010954d

96. L.I. Kioupis, G. Arya, E.J. Maginn, Pressure-enthalpy driven molecular dynamics for thermodynamic property calculation II: applications. Fluid Phase Equilibria 200(1), 93-110 (2002). https://doi. org/10.1016/S0378-3812(02)00015-8

97. J. Vrabec, G.K. Kedia, H. Hasse, Prediction of Joule-Thomson inversion curves for pure fluids and one mixture by molecular simulation. Cryogenics 45(4), 253-258 (2005). https://doi.org/10.1016/j.cryog enics.2004.10.006

98. D.M. Heyes, C.T. Llaguno, Computer simulation and equation of state study of the Boyle and inversion temperature of simple fluids. Chem. Phys. 168(1), 61-68 (1992). https://doi.org/10.1016/03010104(92)80109-9

99. C.M. Colina, E.A. Müller, Molecular simulation of Joule-Thomson inversion curves. Int. J. Thermophys. 20(1), 229-235 (1999). https://doi.org/10.1023/A:1021402902877

100. T.M. Yigzawe, R.J. Sadus, Intermolecular interactions and the thermodynamic properties of supercritical fluids. J. Chem. Phys. 138(19), 194502 (2013). https://doi.org/10.1063/1.4803855

101. R.J. Sadus, Intermolecular potential-based equations of state from molecular simulation and second virial coefficient properties. J. Phys. Chem. B 122(31), 7757-7763 (2018). https://doi.org/10.1021/acs. jpcb.8b05725

Publisher's Note Springer Nature remains neutral with regard to jurisdictional claims in published maps and institutional affiliations. 